\title{
Erratum to: From Dedicated Grid to Volunteer Grid: Large Scale Execution of a Bioinformatics Application
}

\section{Viktors Berstis · Raphaël Bolze • \\ Frédéric Desprez $\cdot$ Kevin Reed}

Published online: 15 October 2009

C) Springer Science + Business Media B.V. 2009

\section{Erratum to: Journal of Grid Computing DOI 10.1007/s10723-009-9130-7}

Unfortunately, the surname of one of the coauthors was misspelled. Instead of "Viktors Bertis" it should read as "Viktors Berstis". The email address was also incorrect. Instead of "bertis@us.ibm.com”, it should read as “berstis@ us.ibm.com".

Also, the affiliation addresses of the authors were updated. Here are the latest contact information.

\section{Berstis}

IBM Systems \& Technology Group,

Austin, TX, USA

e-mail: berstis@us.ibm.com

The online version of the original article can be found at http://dx.doi.org/10.1007/s10723-009-9130-7.

\footnotetext{
V. Berstis

IBM Systems \& Technology Group, Austin, TX, USA

e-mail: berstis@us.ibm.com

R. Bolze $(\varangle) \cdot$ F. Desprez

LIP laboratory, UMR 5668,

CNRS-ENS-Lyon-UCBL-INRIA,

46 allée d'Italie, 69364 Lyon, France

e-mail: raphael.bolze@ens-lyon.fr

F. Desprez

e-mail: frederic.desprez@ens-lyon.fr

K. Reed

IBM Interactive, Chicago, IL, USA

e-mail:knreed@us.ibm.com
}

R. Bolze

LIP laboratory, UMR 5668, CNRS-ENS-Lyon-UCBL-INRIA, 46 allée d'Italie, 69364 Lyon, France e-mail: raphael.bolze@ens-lyon.fr

F. Desprez

LIP laboratory, UMR 5668, CNRS-ENS-Lyon-UCBL-INRIA, 46 allèe d'Italie, 69364 Lyon, France e-mail: frederic.desprez@ens-lyon.fr

K. Reed

IBM Interactive, Chicago, IL, USA e-mail:knreed@us.ibm.com 